\title{
Development of multiplex real-time PCR assays for identification of members of the Anopheles funestus species group Samuel B Vezenegho ${ }^{\dagger 1,2}$, Chris Bass ${ }^{\dagger 3}$, Mirel Puinean ${ }^{3}$, Martin S Williamson ${ }^{3}$, Linda M Field ${ }^{3}$, Maureen Coetzee ${ }^{1,2}$ and Lizette L Koekemoer*1,2
}

\author{
Address: ${ }^{1}$ Vector Control Reference Unit, National Institute for Communicable Diseases of the NHLS, Private Bag X4, Sandringham, Johannesburg \\ 2131, South Africa, ${ }^{2}$ Malaria Entomology Research Unit, School of Pathology of the University of the Witwatersrand and the National Health \\ Laboratory Service, Johannesburg, South Africa and ${ }^{3}$ Department of Biological Chemistry, Rothamsted Research, Harpenden, AL5 2JQ, UK \\ Email: Samuel B Vezenegho - samv@nicd.ac.za; Chris Bass - chris.bass@bbsrc.ac.uk; Mirel Puinean - mirel.puinean@bbsrc.ac.uk; \\ Martin S Williamson - martin.williamson@bbsrc.ac.uk; Linda M Field - lin.field@bbsrc.ac.uk; Maureen Coetzee - maureenc@nicd.ac.za; \\ Lizette L Koekemoer* - lizettek@nicd.ac.za \\ * Corresponding author †Equal contributors
}

Published: 9 December 2009

Malaria Journal 2009, 8:282 doi:10.1 186/1475-2875-8-282
Received: 24 August 2009

Accepted: 9 December 2009

This article is available from: http://www.malariajournal.com/content/8/I/282

(c) 2009 Vezenegho et al; licensee BioMed Central Ltd.

This is an Open Access article distributed under the terms of the Creative Commons Attribution License (http://creativecommons.org/licenses/by/2.0), which permits unrestricted use, distribution, and reproduction in any medium, provided the original work is properly cited.

\begin{abstract}
Background: The malaria vector and non-vector species of the Anopheles funestus group are morphologically very similar and accurate identification is required as part of effective control strategies. In the past, this has relied on morphological and cytogenetic methods but these have been largely superseded by a robust allele-specific PCR (AS-PCR). One disadvantage of AS-PCR is the requirement for post-PCR processing by gel electrophoresis of PCR products. In this study, three new high-throughput 'closed-tube' assays were developed and compared with the previously described AS-PCR technique.
\end{abstract}

Methods: Protocols for three fluorescence-based assays based on Melt Curve Analysis (MCA), High Resolution Melt (HRM) and TaqMan SNP genotyping were developed to detect and discriminate Anopheles parensis, Anopheles leesoni, Anopheles vaneedeni, Anopheles rivulorum and An. funestus s.s. The sensitivity and specificity of these assays were compared with the widely used ASPCR in a blind trial using DNA extracted from wild-caught mosquitoes.

Results: The TaqMan assay proved to be the most sensitive and specific of the three new assays. The MCA and HRM assays initially gave promising results, but were more sensitive to both DNA quality and quantity and consequently showed a higher rate of incorrect identifications.

Conclusion: The TaqMan assay proved to be the most robust of the three protocols tested in this study. This assay very effectively identified all five members of the An. funestus group using fluorescently-labeled probes with distinct emission and excitation spectra allowing their independent detection in a single reaction. This method is at least as sensitive and specific as the gold standard AS-PCR approach and because it has no requirement for post-PCR processing is simpler and more rapid to run. The one disadvantage of the TaqMan assay is the cost of this assay, both in terms of initial capital outlay and running cost per sample, which is higher than AS-PCR. However, the cost of both the real-time PCR machine and fluorescently labelled probes required is falling and in the future the cost of this assay is likely to become closer to that of standard PCR. 


\section{Background}

The Anopheles funestus group consists of five subgroups of mosquitoes namely Anopheles funestus, Anopheles rivulorum, Anopheles minimus, Anopheles aconitus and Anopheles culicifacies and includes one of the most important African vectors of malaria Anopheles funestus s.s. [1-3]. The African mosquito species An. funestus s.s., Anopheles parensis and Anopheles vaneedeni, belonging to the An. funestus subgroup, and Anopheles leesoni and Anopheles rivulorum, belonging to the An. minimus and An. rivulorum subgroups, respectively, are either morphologically identical or very similar and may occur in sympatry over large parts of their distribution [3]. Anopheles funestus s.s., An. rivulorum and An. leesoni have a widespread distribution across sub-Saharan Africa, while An, parensis is found through eastern and southern Africa and An. vaneedeni is restricted to South Africa $[1,2]$.

The vectorial capacity, biology and behaviour of these species also differ. Anopheles funestus s.s. is an efficient vector of the human malaria parasite [2] and this is reflected by its anthropophilic and endophilic behaviour. The other species are predominantly zoophilic and exophilic $[1,2]$ and are thought to have limited, or no, importance as malaria vectors, although An. vaneedeni has been shown to be susceptible to Plasmodium infection under laboratory conditions $[4,5]$, and An. rivulorum has been found infected with the Plasmodium parasite at one locality in Tanzania [6].

Among the members of the An. funestus group, resistance to insecticides has been confirmed only in An. funestus s.s. $[7,8]$. Effective control of this species requires accurate identification methods to discriminate it from the nonvectors and historically this was performed using morphological and cytogenetic methods. Morphological identification utilizes unique characteristics in life stages of the mosquitoes and requires eggs from wild caught females to be reared through the different life forms. This takes about four to six weeks and there is a chance of misidentification because the species within the group show overlapping characteristics [1]. Cytogenetic analysis of giant polytene chromosomes has also been used for species identification after studies revealed species-specific chromosomal banding patterns resulting from fixed paracentric inversions, and published chromosomal maps now exist for An. funestus s.s., An. parensis, An. leesoni and An. rivulorum $[9,10]$. However, this technique involves the extraction of ovarian nurse cells from semi gravid females, thereby limiting its application to this life stage, which means that males and immatures cannot be identified. In addition, An. funestus s.s. can not be discriminated from An. vaneedeni in situations where these species are homozygous for inversions on chromosome arm 2 [10].
Over the last decade these older identification methods have been largely superseded by more rapid DNA-based molecular approaches based on PCR. The first of these was an allele-specific polymerase chain reaction (AS-PCR) assay, which identified An. leesoni, An. vaneedeni, An. rivulorum and An. funestus s.s. by exploiting species-specific polymorphisms in the ribosomal DNA gene (rDNA) [11]. However, this method is unable to distinguish An. parensis from An. vaneedeni and, therefore, an improved AS-PCR protocol was developed to detect and discriminate all five species and this has rapidly become the 'gold standard' for identification of members of the species group [12]. Disadvantages of the current PCR approach include the requirement for post-PCR processing (gel electrophoresis of PCR products) and manual scoring of test samples which can be prone to error due to the similar amplicon sizes generated by certain species.

The aim of the present study was to develop a real-time PCR-based method for identification of members of the An. funestus group that overcomes the disadvantages of the previously described methods and is as sensitive as the gold standard AS-PCR approach. These aims were addressed by developing high-throughput 'closed-tube' approaches based on Melt Curve Analysis (MCA), High Resolution Melt (HRM) and TaqMan SNP genotyping.

\section{Methods}

\section{Samples and DNA extraction}

For the initial optimization of each assay and for the blind trial, field-caught mosquito specimens of An. leesoni, An. vaneedeni, An. rivulorum, An. parensis and An. funestus s.s. were collected from Ghana, South Africa and/or Mozambique. DNA was extracted from single mosquitoes using either the Livak or Collins methods [13,14]. Using these methods DNA yield from a single mosquito was typically 2-5 $\mu \mathrm{g}$ (as determined by absorption at $260 \mathrm{~nm}$ using a NanoDrop spectrophotometer, NanoDrop Technologies). These samples had been initially identified to species at the time of collection using morphology and ASPCR [12]. The blind species identification trial was performed using 96 samples, which included a range of the above species and a number of negative controls. The quality and quantity of DNA obtained from these specimens varied considerably and many had been subject to repeated freeze-thawing so the trial represented a thorough test of the robustness of each assay. To determine the sensitivity of the three identification methods they were further tested using a dilution series of DNA from each of the five species in the An. funestus group. For this, DNA preparations were diluted to $20 \mathrm{ng} / \mu \mathrm{l}$ and then serially diluted down to a 1 in $1 \times 10^{6}$ dilution. 


\section{AS-PCR}

AS-PCR was performed according to the protocol described previously [12] with minor modification. PCR was performed in a final volume of $14 \mu \mathrm{l}$ containing $6 \mu \mathrm{l}$ of ReddyMix PCR master mix (Thermo fisher scientific, UK) and $0.24 \mu \mathrm{M}$ of each primer. The thermal cycling conditions were unchanged.

\section{MCA assay}

The MCA assay utilized the universal forward and speciesspecific reverse primers of Koekemoer et al [12] with the exception of the VAN and FUN primers where new species-specific primers (VAN3 and FUN1) were designed in order to generate amplicons of an optimal melting temperature (Table 1). PCR and subsequent melt curve analysis was carried out using the Rotor-Gene 6000 (Corbett Research). PCR reactions $(20 \mu \mathrm{l})$ consisted of $1 \mu \mathrm{l}$ genomic DNA, $10 \mu \mathrm{l}$ of SensiMix ${ }^{\mathrm{TM}}$ kit (Quantace), $0.4 \mu \mathrm{l}$ SYBR Green 1 (Quantace) and $250 \mathrm{nM}$ of each primer (UV, FUN1, LEES, VAN3, PAR and RIV). Cycling conditions consisted of one cycle of $95^{\circ} \mathrm{C}$ for 10 minutes followed by 40 cycles of $95^{\circ} \mathrm{C}$ for 15 seconds, $55^{\circ} \mathrm{C}$ for 30 seconds and $72^{\circ} \mathrm{C}$ for 30 seconds. This was immediately followed by a melt step of $72-95^{\circ} \mathrm{C}$ rising by $1{ }^{\circ} \mathrm{C}$ and holding for 90 seconds for pre-melt conditioning for the first step and subsequently 5 seconds for each step afterwards. The increase and decrease in fluorescence of SYBR Green during PCR and the melt phase, was acquired on the green channel (470 $\mathrm{nm}$ excitation and 510 emission) of the Rotor-Gene.

\section{HRM}

The HRM assay also utilized the universal forward and species-specific reverse primers of Koekemoer et al [12]. PCR reactions $(25 \mu \mathrm{l})$ contained $12.5 \mu \mathrm{l}$ SensiMix HRM kit (Quantace), $200 \mathrm{nM}$ of each primer; $1 \mu \mathrm{l}$ Eva Green dye, 1 $\mu \mathrm{l}$ genomic DNA. Samples were run on the Rotor-Gene 6000 using the temperature cycling conditions of: 1 cycle of $95^{\circ} \mathrm{C}$ for 10 minutes; 40 cycles of $95^{\circ} \mathrm{C}$ for 15 seconds, $56^{\circ} \mathrm{C}$ for 30 seconds and $72^{\circ} \mathrm{C}$ for 30 second. This was immediately followed by a melt step of $77-94^{\circ} \mathrm{C}$ rising by $0.1^{\circ} \mathrm{C}$ and holding for 90 seconds for pre-melt conditioning for the first step and subsequently 2 seconds for each step afterwards. Changes in fluorescence of Eva Green during PCR and the melt phase were monitored on the green channel (470 $\mathrm{nm}$ excitation and 510 emission) of the Rotor-Gene.

\section{TaqMan}

Nucleotide alignments of ribosomal DNA (rDNA) gene sequences of the different species of An. funestus available in the National Center for Biotechnology Information (NCBI) database revealed species-specific sequences allowing the design of discriminating probes. Unfortunately there was no region of conserved sequence to design a common reverse primer for the TaqMan assay. Therefore the universal forward and species-specific reverse primers of Koekemoer $e t$ al [12] were utilized with the exception of the original primers specific for An. parensis and An. vaneedeni where two new species specific reverse primers were designed (new PAR and VAN1, Table 1). Each species-specific probe was designed to anneal to sequence between the corresponding species-specific reverse primer and the universal forward primer. Probes designed to anneal over sequence containing two or less species-specific SNPs were designed as minor groove binding (MGB) probes (Applied Biosystems) using the Primer Express ${ }^{\mathrm{TM}}$ Software Version 2.0 (Applied Biosystems). The minor groove binder provides more accurate allelic discrimination by increasing the $\mathrm{T}_{\mathrm{M}}$ between matched and mis-matched probes [15]. Probes designed to anneal over sequence containing more than two spe-

Table I: Primers and probes used in the identification of Anopheles funestus species using real time assays (MCA, HRM and TaqMan)

\begin{tabular}{|c|c|c|c|c|}
\hline Name & $\begin{array}{l}\text { Primer } \\
\text { /probe }\end{array}$ & Reporter Dye & Sequence (5'-3') & Quencher \\
\hline FUNI & Primer & - & GGCATCGATGGGTTAATCATG & - \\
\hline VANI & Primer & - & AAACCCCAAGATGTGCTCC & - \\
\hline VAN3 & primer & - & GGTTTTCAAATGAATCTC & - \\
\hline N PAR & Primer & - & ATACTTGTGTGTGTGTGTATTTG & - \\
\hline RIV TM & probe & Cy5 & CTATGGCGAGACCCCGTCTAGTG & $\mathrm{BHQ}^{\mathrm{a}}$ \\
\hline FUN TM & probe & ROX & CATGGGGAAATTCAATCGAAAACCTCT & $\mathrm{BHQ}^{\mathrm{a}}$ \\
\hline PAR TM & probe & VIC & CGG AAC CTA GCT TGG & MGBNFQb \\
\hline VAN TM & probe & Quasar 705 & CGT TGT GAA AAA TGG AGA TTC ATT TGA AAA CC & $\mathrm{BHQ}^{\mathrm{a}}$ \\
\hline LEES TM & probe & 6-FAM & CCG ACC GAT GTA CA & MGBNFQb \\
\hline
\end{tabular}


cies-specific SNPs were designed manually and synthesized as dual-labeled probes by Thermo Fisher Scientific or Biosearch Technologies. The sequence and quencher modifications of each probe are shown in Table 1 . The five probes were labeled at the $5^{\prime}$ end with VIC (probe PAR TM) for An. parensis detection, 6FAM (probe LEE TM) for An. leesoni detection, Cy5 (probe RIV TM) for An. rivulorum detection, ROX (probe FUN TM) for An. funestus s.s. detection and Quasar 705 (probe VAN TM) for An. vaneedeni detection. These five flurophores have distinct emission and excitation spectra allowing their independent detection in a single reaction.

PCR reactions $(20 \mu \mathrm{l})$ contained $10 \mu \mathrm{l}$ of SensiMix DNA kit (Quantace), $200 \mathrm{nM}$ of each probe, $1 \mu \mathrm{M}$ each of UV, FUN and VAN 1 primers, $0.5 \mu \mathrm{M}$ of RIV, LEES, New PAR primers and $1 \mu \mathrm{l}$ of DNA template. Reactions were run on the Rotor-Gene 6000 using the temperature cycling conditions of: 10 minutes at $95^{\circ} \mathrm{C}$ followed by 35 cycles of $95^{\circ} \mathrm{C}$ for 15 seconds and $60^{\circ} \mathrm{C}$ for 60 seconds. The increase in VIC, FAM, ROX, CY5 and Quasar 705 fluorescence was monitored in real time by acquiring each cycle on the yellow (530 $\mathrm{nm}$ excitation and $555 \mathrm{~nm}$ emission), green ( $470 \mathrm{~nm}$ excitation and 510 emission), orange (585 $\mathrm{nm}$ excitation and $610 \mathrm{~nm}$ emission), red (625 nm excitation and $660 \mathrm{~nm}$ emission) and crimson channels (680 $\mathrm{nm}$ excitation and $710 \mathrm{~nm}$ emission) of the Rotor-Gene respectively.

\section{Results}

\section{AS-PCR}

The sensitivity of the AS-PCR assay was evaluated using serially diluted DNAs from each species of mosquito. The detection limit of each of the five species was a 1 in 500 dilution, which represents 0.04 ng of DNA. The results from the blind species identification trial using the PCR method are shown in Table 2. The AS-PCR method showed a low failure rate (no amplification in PCR) and did not incorrectly identify any samples.

\section{MCA assay}

The melt-curve real-time PCR assay uses allele-specific primers to generate products of different length and/or GC content that are discriminated by the different melt temperature of the PCR amplicons. PCR in the presence of the intercalating dye SYBR green is followed by a melt step

Table 2: Performance of four assays in the Anopheles funestus group species identification blind trial

\begin{tabular}{lcccc}
\hline & AS-PCR & MCA & HRM & TaqMan \\
\hline Correct scores & 90 & 84 & 88 & 89 \\
Failed reactions & 6 & 4 & 2 & 7 \\
Miscored & 0 & 8 & 6 & 0 \\
\hline
\end{tabular}

which results in denaturisation of the PCR products and a resulting decrease in SYBR green fluorescence as the dye is released. A plot of the negative first derivative of the collected fluorescence against temperature results in melt peaks with characteristic melting temperatures $\left(T_{M}\right)$.

After optimization using DNA templates of known species the MCA was able to detect and discriminate An. leesoni, An. vaneedeni, An. rivulorum, An. parensis and An. funestus s.s. Figure 1 depicts the characteristic melt curves produced by the five different mosquito species after plotting a negative first derivative of fluorescence against temperature. The average $T_{M}$ for 10 samples per species was determined and the results are presented in Table 3. During optimization it became clear that it was sometimes difficult to differentiate An. vaneedeni from An. parensis and An. funestus s.s. specimens as the melt-temperatures of the amplicons produced from each species was similar. Attempts were made to improve the discrimination of these samples by designing alternative primers (to generate smaller or larger amplicons with different $T_{M}$ ) for the detection of An. vaneedeni and An. funestus s.s. but the primers described in the methods ultimately gave the clearest discrimination of these species. This issue affected the performance of this assay in the species identification trial (Table 2) where the MCA exhibited a low failure rate but incorrectly identified a number of samples. In most instances this was due to misidentification of An. vaneedeni, An. funestus s.s. and An. parensis specimens. Analytical sensitivity of the MCA assay was investigated using a dilution series of DNAs for each of the five species and the detection limit was found to be a 1 in a 1,000 dilution, which represents $0.02 \mathrm{ng}$ of DNA.

\section{HRM}

High Resolution Melt (HRM) is an extension of the MCA assay but employs next generation real-time PCR thermocyclers with high thermal and optical precision. HRM analyses involve the PCR amplification of DNA containing the SNP(s) of interest in the presence of a third generation fluorescent dsDNA dye. The new generation of dyes for this purpose such as SYTO 9 (Invitrogen), LC Green (Idaho Technologies) and Eva Green (Biotium Inc) are less inhibitory to PCR than traditional dyes, which allow them to be used at higher concentration to achieve maxi-

Table 3: Average melt curve $T_{M}$ and standard deviation values for different members of the An. funestus group.

\begin{tabular}{ccc}
\hline Species & Average $\mathbf{T}_{\mathbf{M}}$ & Standard deviation ( $\mathbf{\pm})$ \\
\hline An. rivulorum & 88.71 & 0.087 \\
An. Leesoni & 84.69 & 0.34 \\
An. vaneedeni & 86.17 & 0.6 \\
An. Funestus & 87 & 0 \\
An. Parensis & 85.82 & 0.175
\end{tabular}




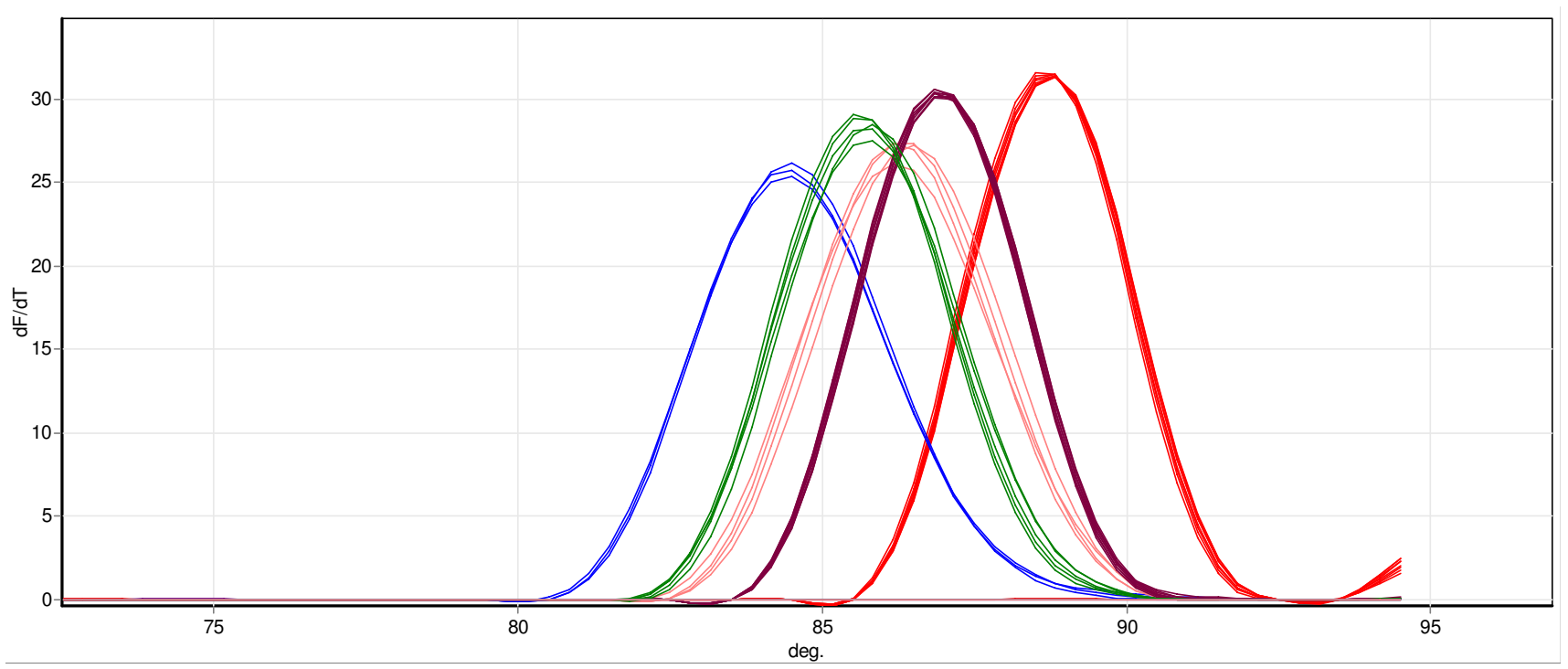

Figure I

Identification of members of the An. funestus group by melt-curve real-time PCR analysis. In this example five to seven specimens of An. rivulorum (red trace), An. leesoni (blue trace), An. funestus (brown trace), An. parensis (green trace) and An. vaneedeni (pink trace) were tested. A plot of negative first derivative of the collected fluorescence against temperature results in melt peaks with characteristic melting temperatures $\left(T_{M}\right)$.

mum saturation of the resulting dsDNA amplicon. A highresolution melt step is then performed, centered around the $\mathrm{T}_{\mathrm{M}}$ of the amplicon. As the dsDNA dissociates into single strands the dye is released and the fluorescence diminishes giving a melt curve profile characteristic of the sequence of the amplicon [16].

After optimization the HRM assay was able to discriminate all five target species of the An. funestus group from the melting temperature of the PCR amplicons which ranged in size from 146 to $587 \mathrm{bp}$. Samples were scored by examining normalized and difference melt plots using the associated Rotor-Gene Software (version 1.7). Figure 2 , shows a normalized plot as well as a difference plot for several specimens of each species of mosquito. The difference plot generated from the normalized data highlights differences between a selected genotype and the other samples. The sensitivity of the HRM assay was evaluated using a dilution series of DNA for each species. The detection limit of each of the five species was a 1 in a 1,000 dilution, which represents 0.02 ng of DNA. When the performance of the HRM assay was assessed in a blind trial using DNA extracted from wild-caught mosquitoes (Table 2) it displayed a low failure rate but like the MCA assay incorrectly identified a number of samples. This included the misidentification of two water negative controls (as An. funestus s.s. and An. rivulorum), which may indicate a potential problem with background/non-specific fluorescence in this assay.

\section{TaqMan}

The TaqMan assay is a PCR method which uses oligonucleotide probes that are dual-labeled with a fluorescent reporter dye and a quencher molecule. Amplification of the probe-specific product causes cleavage of the probe, generating an increase in reporter fluorescence as the reporter dye is released away from the quencher. By using different reporter dyes, cleavage of allele-specific probes can be detected in a single PCR.

After optimization the multiplex TaqMan assay effectively identified control templates of the five members of the An. funestus group. Figure 3 shows an example of using this assay for the identification of seven to ten samples of each species. An increase in the fluorescence of Cy5 (probe RIV), 6FAM (LEES probe), ROX (FUN probe), VIC (PAR probe) and Quasar 705 (VAN probe) identifies An. rivulorum, An. leesoni, An. funestus s.s., An. parensis and An. vaneedeni specimens respectively. An increase in two or more of the dyes would indicate a hybrid or a contaminated sample. During optimization it was noticed that the fluorescent signal generated by specific binding of the VAN probe was significantly lower than the other probes as quantified in relative fluorescent units. The exact reason(s) for this is unknown, however, amplification of the specific An. vaneedeni amplicon was acceptable (when checked by running products on agarose gel electrophoresis) and replacement of the fluorescent dye label and/or redesign of the probe did not improve the fluorescent sig- 

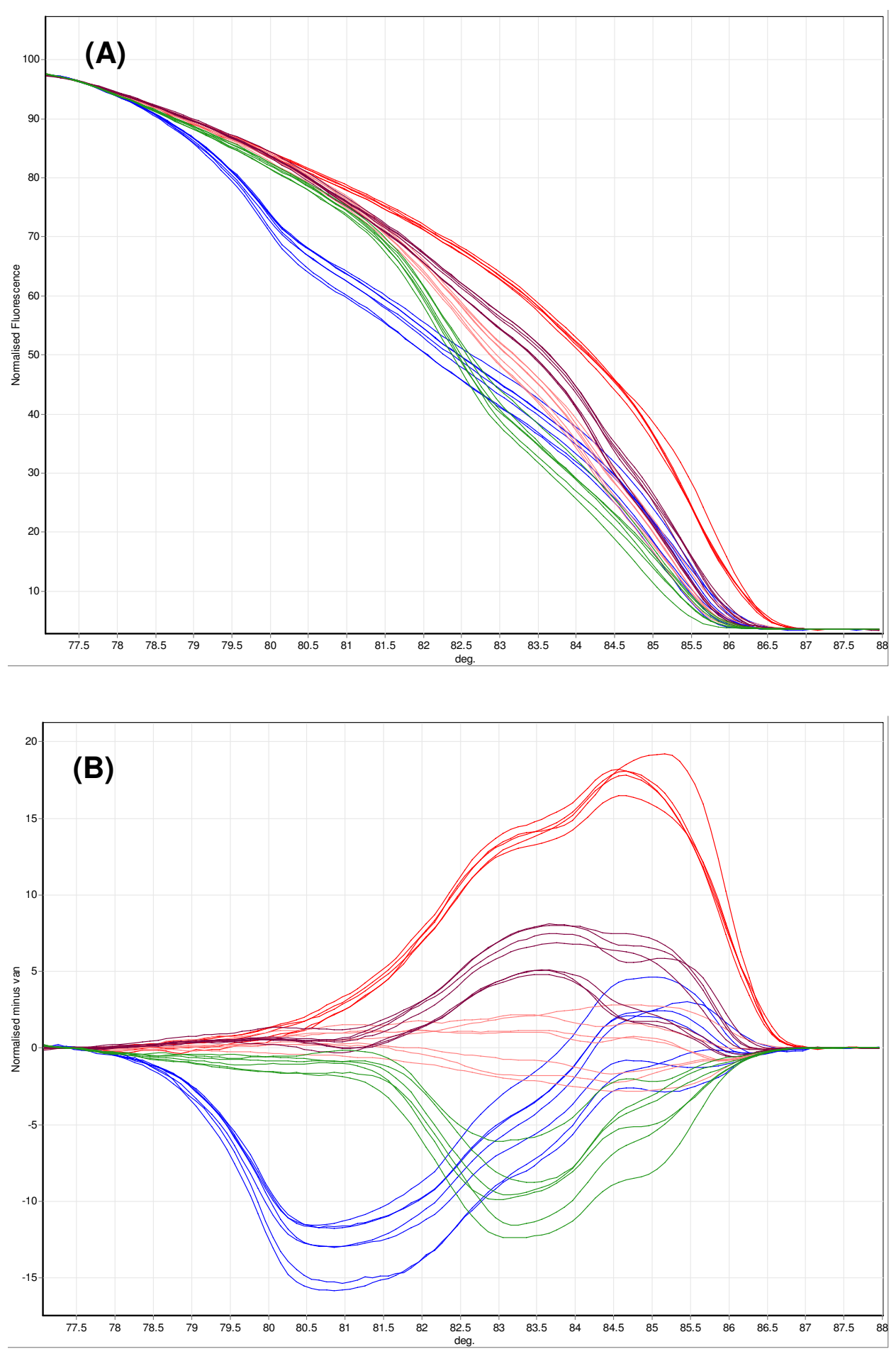

\section{Figure 2}

Identification of members of the An. funestus group by High Resolution Melt analysis. In this example, five to seven specimens of An. rivulorum (red trace), An. leesoni (blue trace), An. funestus (brown trace), An. parensis (green trace) and An. vaneedeni (pink trace) were tested. A) Normalized melt curve for different An. funestus species. B) Difference plot for samples as in $(\mathrm{A})$. 

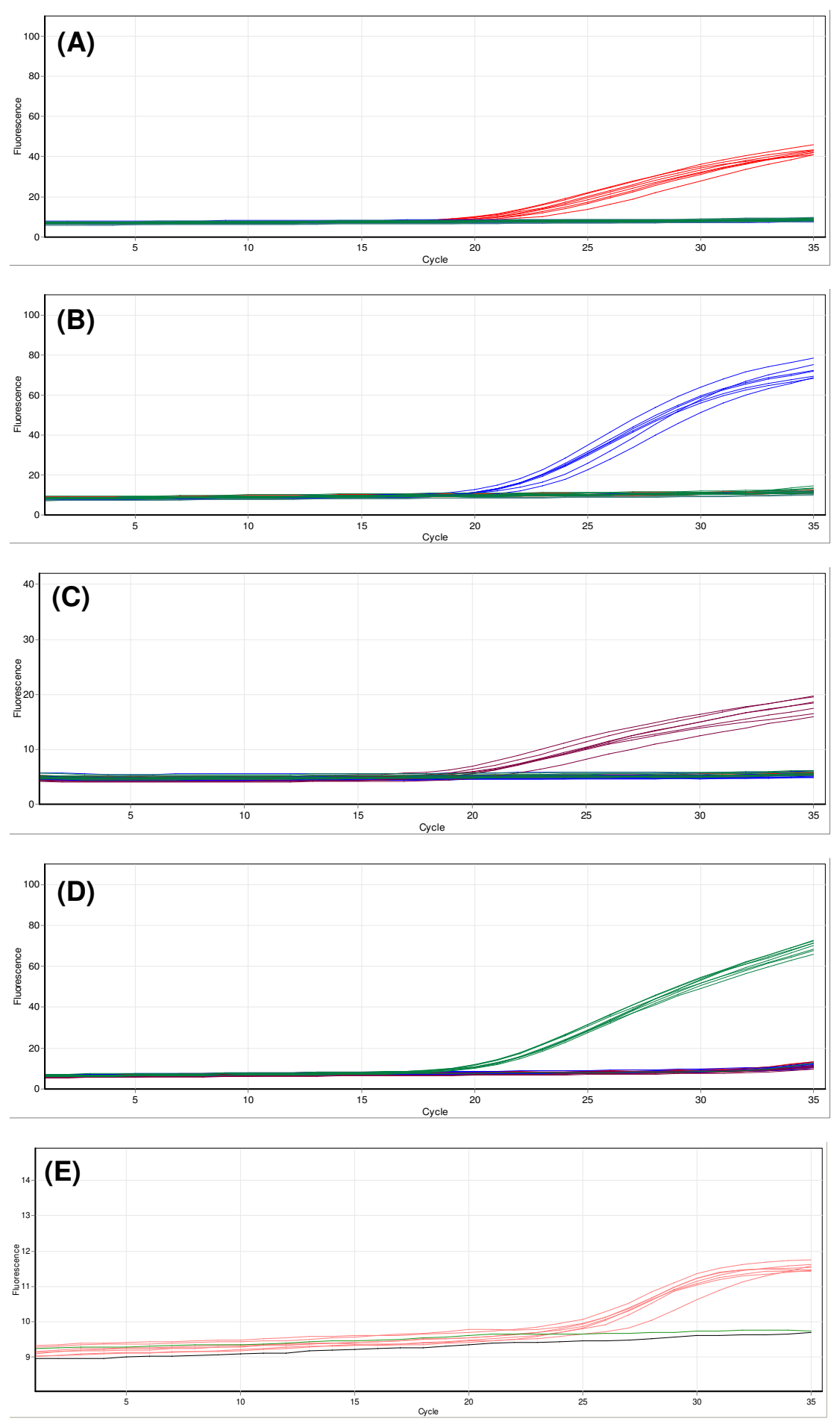

Figure 3

Identification of members of the An. funestus group using the multiplex TaqMan assay. Seven to ten specimens of An. rivulorum (red trace), An. leesoni (blue trace), An. funestus (brown trace), An. parensis (green trace) and An. vaneedeni (pink trace) were tested. (A) Cycling of the RIV probe (Cy5 labelled), (B) cycling of the LEES probe (6FAM labelled), (C) cycling of the FUN probe (ROX labelled), (D) cycling of the PAR probe (VIC labelled) and (E) cycling of the van probe (Quasar 705 labelled). 
nal generated. Despite the lower fluorescent signal it was still possible to effectively identify An. vaneedeni specimens by using the auto-scale function of the associated Rotor-Gene software. The TaqMan PCR was originally run for 40 temperature cycles, however, low level non-specific amplification was occasionally observed from cycling of the LEES (6FAM-labelled) and PAR (VIC-labelled) probes specific for An. leesoni and An. parensis respectively after 35 cycles. This was successfully eliminated by restricting the number of temperature cycles to 35 .

The analytical sensitivity of the TaqMan assay was assessed using serially diluted DNAs from the five representative species. The detection limit of each species was a 1 in a 1,000 dilution, which represents 0.02 ng of DNA. The performance of the TaqMan assay in the blind trial using DNA extracted from wild-caught mosquitoes (Table 2) was found to be comparable to AS-PCR with a low failure rate and no incorrectly identified samples.

\section{Discussion}

In this study, protocols were developed for three highthroughput multiplex real-time PCR assays (TaqMan, HRM and MCA) for the identification of members of the An. funestus group that occur commonly throughout much of Africa. Unlike conventional AS-PCR, these assays do not require processing of samples by agarose gel electrophoresis, which is time consuming, restricts throughput and requires the use of the safety hazard ethidium bromide.

Of the three fluorescent assays, the MCA assay held promise for being the most cost-effective as it employs standard oligonucleotide primers, has no requirement for fluorescently labelled probes and can be run on single channel real-time PCR machines. Analysis of one sample costs approximately US $\$ 0.65$ using this method. The assay developed in this study was able to detect and discriminate all five target species using DNA templates of known concentration and was more sensitive than the standard AS-PCR. However, when the assay was tested in a blind trial using DNA extracted from wild-caught mosquitoes of variable concentration and quality it became evident that this assay occasionally misidentifies An. vaneedeni, An. funestus s.s. and An. parensis specimens. This is likely due to the relatively close $\mathrm{T}_{\mathrm{M}}$ of the specific amplicons produced during amplification of each species (Table 3). For MCA it is recommended that the amount of DNA template used in PCR is consistent between samples as large differences in starting template will affect the observed $T_{M}$. It is, therefore, possible that this assay could be improved if DNA concentration was adjusted. However this constitutes an additional step in the protocol and would require DNA quantification using a spectrophotometer or gel electrophoresis. It is likely that the chances of misidentifi- cation will be reduced when the assay is used to test mosquitoes collected from much of Sub-Saharan Africa where An. vaneedeni does not occur due to the limited distribution of this species. This should make scoring the other species using MCA easier as the gap in $\mathrm{T}_{\mathrm{M}}$ between amplicons generated from An. funestus s.s. and An. parensis specimens will be clearer.

To see if a more recent melt-curve approach could more accurately identify the small difference in $T_{M}$ between these three species an assay based on HRM was developed. Like MCA the running cost of HRM is low (US $\$ 0.65$ per sample) as it uses standard oligonucleotide primers and a cheap intercalating dye; however the disadvantage of this platform is the capital cost required for the more expensive real-time PCR machine required. The HRM method showed promise during optimization with templates of known genotype (where DNA concentration was adjusted to be consistent for all samples) with a sensitivity greater than AS-PCR, but, like the MCA assay, subsequently performed less well in the blind genotyping trial. This is likely explained by variable DNA quality and quantity among the samples tested, leading to some samples amplifying after $\sim 30$ cycles or failing to reach full plateau phase. In addition the HRM method incorrectly identified two negative control samples as An. leesoni and An. rivulorum indicating a potential problem with background/non-specific fluorescence.

In order to overcome the problems with the two meltcurve based approaches, an assay based on TaqMan SNP genotyping was developed. This approach has proven to be very sensitive and robust in the detection and discrimination of the Plasmodium species responsible for human malaria, members of the An. gambiae complex that vector the disease and for detection of mutations in the mosquito genome that confer insecticide resistance [17-20]. In tests of analytical sensitivity and in the blind trial the TaqMan assay was shown to have a specificity and sensitivity at least as good as the standard AS-PCR. Of the three fluorescence assays tested in this study, this assay was the only method to record no misidentification of samples indicating the robustness of this platform. The unambiguous identification of samples by the TaqMan assay may in part be due to the dual layer of specificity provided by the combination of both allele-specific primers and allele-specific probes. The running cost of the TaqMan assay, as performed in this study, is slightly higher than MCA and HRM as it uses fluorescently-labelled probes (US \$0.95 per sample). Additional experiments were carried out to see if reagent volumes could be reduced without affecting the sensitivity of the assay. With the real-time PCR machine used in this study (Rotor-Gene 6000 ${ }^{\mathrm{Tm}}$, Corbett Research) no loss of sensitivity was observed for half volumes of reagents which reduces the running cost to US 
$\$ 0.55$ per sample. In addition, to reduce the costs further the end-user may wish to consider selecting which probes to use in PCR based on the area where the mosquito specimens to be tested are collected, for example, it may not be necessary to test for An. vaneedeni in many Sub-Saharan countries as its distribution is limited to a localized region in northern South Africa. The one disadvantage of the TaqMan assay is the initial cost of the real-time PCR machine required [18]. However, the price of real-time PCR machines are falling and in the future is likely to become closer to standard thermocyclers.

\section{Conclusion}

Of the new assays tested in this study, the TaqMan assay proved to be the most robust. The assay uses fluorescentlylabeled probes with distinct emission and excitation spectra allowing the independent detection of five members of the An. funestus group in a single reaction. This method is at least as sensitive and specific as the gold standard ASPCR approach and because it has no requirement for postPCR processing is simpler to run and capable of higher throughput.

\section{Competing interests}

The authors declare that they have no competing interests.

\section{Authors' contributions}

SBV helped to design the assays, optimized and tested all the assays and helped draft the manuscript. CB designed the assays and drafted the manuscript. MP helped to design, optimize and test the TaqMan assay and helped review the manuscript. MSW, LMF, MC, helped draft and critically review the manuscript. LLK conceived the project and helped draft and critically review the manuscript. All authors read and approved the final manuscript.

\section{Acknowledgements}

We thank the Mpumalanga Malaria Control Programme for supplying fieldcollected mosquitoes from South Africa, Prof Richard Hunt for samples from Ghana; Varsha Ramdeen and Mike Coleman for mosquitoes from Mozambique. This work was supported by a National Research Foundation, South Africa, grant to LLK and the NRF/DST Research Chair Initiative grant to $M C$, as well as the Innovative Vector Control Consortium. Rothamsted Research receives grant aided support from the Biotechnology and Biological Sciences Research Council of the UK.

\section{References}

I. Gillies MT, Coetzee M: Supplement to the Anophelinae of Africa South of the Sahara. Publications of the South African Institute for Medical Research, No 551987.

2. Gillies MT, De Meillon B: The Anophelinae of Africa south of the Sahara. Publications of the South African Institute for Medical Research, No 541968.

3. Harbach RE: The classification of the genus Anopheles (Diptera: Culicidae): a working hypothesis of phylogenetic relationships. Bull Entomol Res 2004, 94:537-553.

4. Coetzee M, Fontenille D: Advances in the study of Anopheles funestus, a major vector of malaria in Africa. Insect Biochem Mol Biol 2004, 34:599-605.
5. De Meillon B, Van Eeden G, Coetzee L, Coetzee M, Meiswinkel R, Du Troit C, Hansford C: Observations on a species of Anopheles funestus subgroup, a suspected exophilic vector of malaria parasites in north-eastern Transvaal, South Africa. Mosq News 1977, 37:657-66I.

6. Wilkes TJ, Matola YG, Charlwood JD: Anopheles rivulorum, a vector of human malaria in Africa. Med Vet Entomol 1996, 10:108-110.

7. Brooke BD, Kloke G, Hunt RH: Bioassay and biochemical analyses of insecticide resistance in Southern African Anopheles (Diptera: Culicidae). Bull Entomol Res 200I, 91:265-272.

8. Hargreaves K, Koekemoer LL, Brooke BD, Hunt RH, Mthembu J, Coetzee M: Anopheles funestus is resistant to pyrethroid insecticides in South Africa. Med Vet Entomol 2000, 14:181-189.

9. Green CA: Cladistic analysis of mosquito chromosome data (Anopheles (Cellia) Myzomyia). J Hered I982, 73:3-II.

10. Green CA, Hunt RH: Interpretation of variation in ovarian polytene chromosomes of Anopheles funestus Giles, A. parensis Gillies and A. aruni? Genetica 1980, 51 : I87-198.

II. Koekemoer LL, Lochouarn LL, Hunt RH, Coetzee M: Single-strand conformation polymorphism analysis for identification of four members of the Anopheles funestus (Diptera: Culicidae) group. J Med Entomol 1999, 36:125-130.

12. Koekemoer LL, Kamau L, Hunt RH, Coetzee M: Cocktail polymerase chain reaction assay to identify members of the Anopheles funestus (Diptera: Culicidae) group. Am J Top Med Hyg 2002, 66:804-8II.

13. Collins F, Mendez AM, Rasmussen MO, Mehaffey CF, Besansky NJ, Finnerty V: A ribosomal RNA gene probe differentiates member species of the Anopheles gambiae complex. Am J Trop Med Hyg 1987, 37:37-4I.

14. Livak KJ: Organization and mapping of a sequence on the Drosophila melanogaster $X$-chromosome and $Y$-chromosome that is transcribed during spermatogenesis. Genetics 1984, 107:6 I I-634.

15. Afonina I, Zivarts M, Kutyavin I, Lukhtanov E, Gamper H, Meyer RB: Efficient priming of PCR with short oligonucleotides conjugated to a minor groove binder. Nucleic Acids Res 1997, 25:2657-2660.

16. Liew M, Pryor R, Palais R, Meadows C, Erali M, Lyon E, Wittwer C: Genotyping of single-nucleotide polymorphisms by high-resolution melting of small amplicons. Clin Chem 2004, 50:1 I 156-1164.

17. Bass C, Nikou D, Blagborough AM, Vontas J, Sinden RE, Williamson MS, Field LM: PCR-based detection of Plasmodium in Anopheles mosquitoes: a comparison of a new high-throughput assay with existing methods. Malar J 2008, 7:177.

18. Bass C, Nikou D, Donnelly MJ, Williamson MS, Ranson H, Ball A, Vontas J, Field LM: Detection of knockdown resistance (kdr) mutations in Anopheles gambiae: a comparison of two new highthroughput assays with existing methods. Malar J 2007, 6: I I I.

19. Bass C, Williamson MS, Field LM: Development of a multiplex real-time PCR assay for identification of members of the Anopheles gambiae s pecies complex. Acta Trop 2008, 107:50-53.

20. Bass C, Williamson MS, Wilding CS, Donnelly MJ, Field LM: Identification of the main malaria vectors in the Anopheles gambiae species complex using a TaqMan real-time PCR assay. Malar J 2007, 6:155.

Publish with Bio Med Central and every scientist can read your work free of charge

"BioMed Central will be the most significant development for disseminating the results of biomedical research in our lifetime. "

Sir Paul Nurse, Cancer Research UK

Your research papers will be:

- available free of charge to the entire biomedical community

- peer reviewed and published immediately upon acceptance

- cited in PubMed and archived on PubMed Central

- yours - you keep the copyright 